\title{
Hacia una taxonomía constructiva de las tapias de tierra y fábricas encofradas históricas
}

\section{Towards a constructive taxonomy of the historical rammed-earth and formwork masonry}

$\underline{\text { I. J. Gil-Crespo }}^{(*)}$, L. Maldonado-Ramos ${ }^{(*)}$

\section{RESUMEN}

El objetivo fundamental de la investigación es el estudio de los fundamentos constructivos de las fábricas históricas de tapia de tierra y mampostería encofrada de las fortificaciones bajomedievales. Ante las incertidumbres detectadas en la datación de estas estructuras y frente al excesivo número de excepciones que quedan fuera de las clasificaciones tradicionalmente empleadas para estudiar este tipo de técnicas, el artículo desarrolla una propuesta de tipología o taxonomía constructiva basada en un sistema abierto. Este sistema nace del análisis de un número significativo de fábricas y atiende a diversos parámetros de control para la completa caracterización de cada fábrica: material, grado de compactación, función constructiva, encofrado, acabado superficial, combinación de materiales y situación, profundidad, formación y sección de los agujales. Cada fábrica se clasifica mediante una etiqueta alfanumérica. El sistema permite establecer tipos constructivos con los que se genera una clasificación cronotipológica constructiva.

Palabras clave: Tapia de tierra; tapia de cal y canto; historia de la construcción; clasificación constructiva.

\section{ABSTRACT}

The principal aim of the research is the study of the constructive reasons of the historical masonries built with rammed earth and formwork masonry, belonging to late medieval fortifications. Uncertainties have been warned about dating the historical masonries and there are excessive exceptions that do not fall in the rankings traditionally employed to study these techniques. Due to these reasons, the article develops a proposal of constructive typology or taxonomy based in an open system that comes from the analysis of a representative number of masonries and that attends to several control parameters tor the complete characterization of each masonry: material, compaction degree, constructive function, formwork, rendering, combination of materials and, last, the situation, deepness, formation and section of the putlogholes. Each masonry is classified through an alphanumeric label. The system allows establishing several constructive types which it is possible to do a cronotypological and constructive classification.

Keywords: Rammed-earth; masonry formwork; construction history; constructive classification.

(*) Escuela Técnica Superior de Arquitectura - Universidad Politécnica de Madrid (España).

Persona de contacto/Corresponding author: ignaciojaviergilcrespo@gmail.com (I. J. Gil-Crespo)

Cómo citar este artículo/Citation: Gil-Crespo, I. J., Maldonado-Ramos, L. (2015). Hacia una taxonomía constructiva de las tapias de tierra y fábricas encofradas históricas. Informes de la Construcción, 67(538): eo86, doi: http://dx.doi.org/10.3989/ic.14.009.

Licencia/License: Salvo indicación contraria, todos los contenidos de la edición electrónica de Informes de la Construcción se distribuyen bajo una licencia de uso y distribución Creative Commons Reconocimiento no Comercial 3.o. España (cc-by-nc). 


\section{OBJETIVOS Y MÉTODO}

El artículo tiene por objetivo principal exponer una serie de parámetros de control que permitan analizar las fábricas históricas de tapia de tierra y de cal y canto desde una visión global de sus aspectos morfológicos y constructivos. La aplicación metodológica de estos parámetros en el estudio de las fábricas históricas encofradas, sirven para definir la base de la propuesta para una clasificación taxonómica y cronotipológica de estas fábricas.

En los estudios historiográficos y artísticos se ha detectado una incertidumbre e incorrecciones en la datación de algunas construcciones fortificadas en las que no hay elementos documentales suficientes para datar su construcción. De igual manera, ante la falta de elementos artísticos o estilísticos en unos sobrios edificios eminentemente funcionales se ha recurrido a considerar los aspectos constructivos como referentes cronológicos (1) (Figura 1). El estudio constructivo comparado de las fábricas puede arrojar información verosímil sobre su época de construcción y permite elaborar hipótesis parciales sobre la datación de cada caso de estudio, que se corroborarán con un posterior análisis documental bibliográfico-archivístico y con el apoyo de métodos de caracterización y datación físico-química o de prospección arqueológica paralela que extraiga objetos coetáneos a la construcción.

La investigación original de la que este artículo extrae y desarrolla las conclusiones se circunscribe al ámbito territorial de la provincia de Soria, donde se han documentado las fortificaciones fronterizas construidas durante la Baja Edad Media ${ }^{1}$.Con el fin de estudiar sus fundamentos constructivos y para establecer unos parámetros de control y comparación que permitan estudiar metódicamente las fábricas históricas, se ha realizado una investigación paralela con una exhaustiva toma de datos en 89 fortificaciones distribuidas entre España y Portugal, de las que 43 están construidas total o parcialmente con tapia de tierra o mampostería encofrada. Si bien la distribución geográfica de estos casos no abarca todas las regiones donde hay fábricas encofradas históricas -razón por la que hay que considerar la provisionalidad de los resultados de esta propuesta-, se han considerado diversas regiones representativas como son el ámbito castellano (provincias de Soria, Segovia, Palencia, Toledo y Madrid), el andaluz (Almería, Granada y Sevilla) y el sur de Portugal (Algarve). De otras regiones tradicionalmente prolijas en fábricas de tapias de tierra o de cal y canto, como son el ámbito valenciano y la frontera histórica entre León y Castilla, los datos que no se han podido tomar in situ han sido tomados de las diversas investigaciones publicadas.

Se han recogido los datos referidos a las dimensiones constructivas de la fábrica de tapia: la altura de los niveles constructivos, la longitud de los cajones, el talud entre juntas constructivas, la distancia entre mechinales y las dimensiones - anchura, altura y profundidad - del mechinal, además de aspectos formales y constitutivos, como el sistema de for-

\footnotetext{
${ }^{1}$ La investigación se extrae de la tesis doctoral «Fundamentos constructivos de las fortificaciones fronterizas entre las coronas de Castilla y Aragón de los siglos XII al XV en la actual provincia de Soria» de Ignacio Javier Gil Crespo leída en la ETS de Arquitectura de Madrid, siendo los directores los profesores Santiago Huerta y Luis Maldonado (2).
}

mación del mechinal, la lectura de las huellas de los tapiales y piezas del encofrado en la superficie de la tapia, el acabado superficial, la combinación con otros materiales o la función constructiva de la tapia respecto al conjunto del elemento arquitectónico.

La definición del problema objeto de la investigación surge de la falta de análisis metódico y ordenado del sistema fortificado bajomedieval, así como por las diversas dataciones erróneas o sin fundamentar, derivado de la aparente falta de fuentes documentales sobre la fundación o las reparaciones de las fortificaciones. La datación ha sido dependiente de la Historia del Arte y del estilo; sin embargo, estos edificios carecen de elementos estilísticos que permitan fundamentar una datación por esta vía. Este mismo problema se presenta en cuando se aborda el análisis constructivo de la arquitectura tradicional (3). Por tanto, se debe generar un estudio tipológico de los aspectos constructivos que garantice una puesta en paralelo con fiabilidad y eficacia para establecer conclusiones cronológicas y siempre desde el estudio constructivo.

A pesar de que el patrimonio castrense está protegido bajo el amparo legal, distintas circunstancias históricas han favorecido su abandono, ruina y explotación como cantera para otras construcciones cercanas. La reciente puesta en valor de estos edificios se refleja en la aprobación del Plan Nacional de Castillos, la actualización del inventario de fortificaciones españolas (4), la redacción de la Carta de Baños de la Encina - cuyas recomendaciones metodológicas ha seguido esta investigación-y la elaboración de planes estratégicos (5) con el fin de documentar, analizar y conservar tan vasto patrimonio.

La necesidad de un conocimiento profundo y detallado del amplísimo patrimonio castrense medieval requeriría de un gasto económico importante si se plantean los sistemas de datación de edificios históricos basados en la recogida selectiva de muestras y su ensayo en laboratorio para determinar su antigüedad mediante métodos como el radiocarbono o la dendrocronología. El abandono al que están sometidos está provocando su progresiva ruina y no parece haber esperanzas de reutilización, máxime cuando las poblaciones a las que en un pasado protegieron sufren un acelerado envejecimiento y abandono demográfico. Por estos motivos se hace necesario un estudio sistemático que plantee de forma asequible -en cuanto a coste de medios y dispendio económico- un método de análisis y conocimiento de los fundamentos constructivos de la construcción castrense bajomedieval.

Con esta revisión y la puesta en común con la comunidad científica que está realizando análisis similares para otros conjuntos de edificios a través de diversas vías de comunicación científica se debe llegar a establecer una cronotipología constructiva en la que se reconozcan las técnicas constructivas a lo largo de la historia con el fin de que cualquier edificio o sus partes puedan compararse y datarse en función de esta secuenciación contrastada en base al análisis de sus fábricas.

El método cronotipológico consta en la fijación de unos hitos o indicadores cronotipológicos de cada época dado que los aspectos constructivos tienden a presentar un progresión temporal continua, atendiendo tanto a los episodios de arcaísmo local como a las características de uniformidad ambiental (6). 

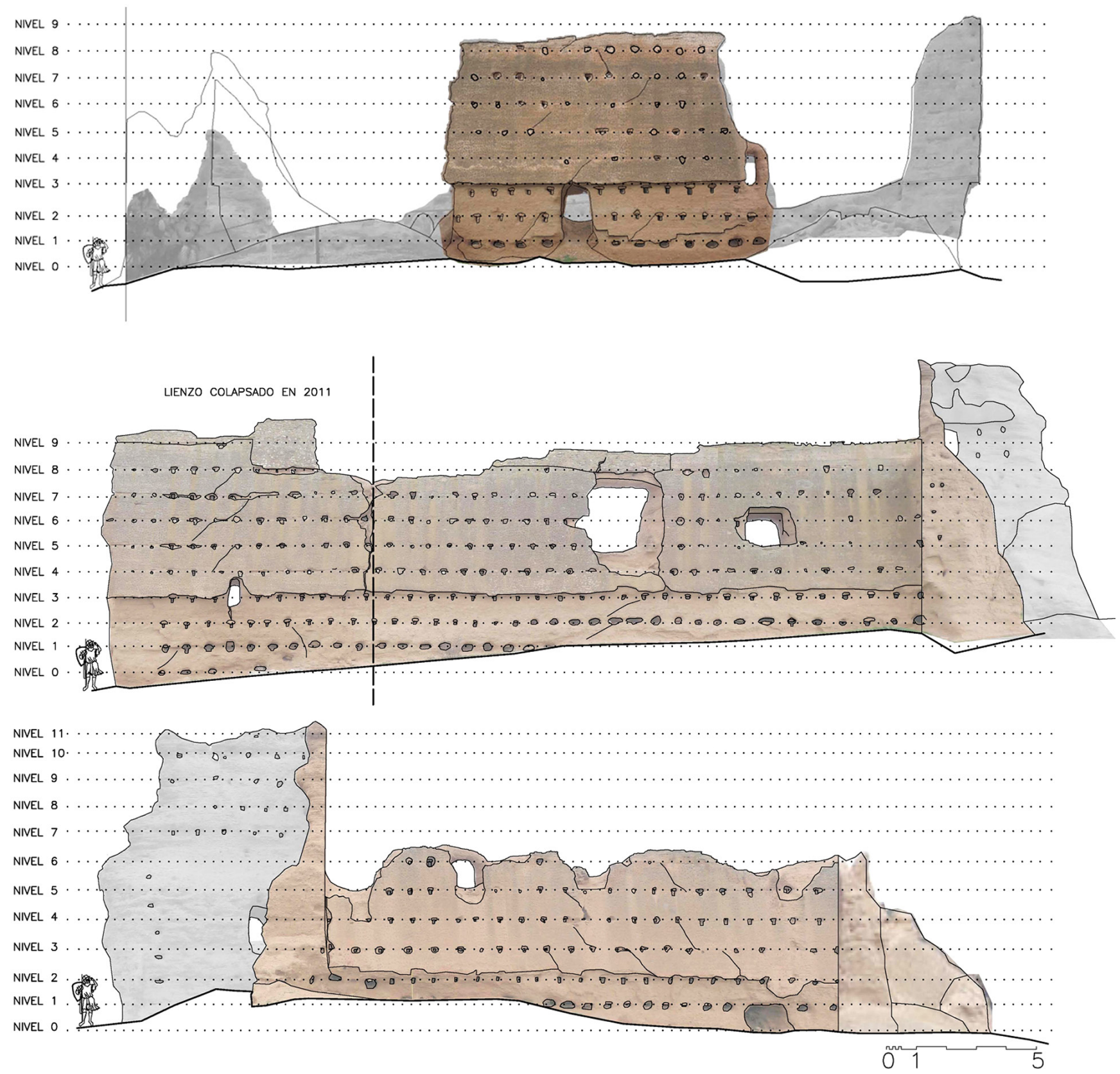

Figura 1. Alzados del castillo de Serón de Nágima (Soria), caso significativo que la tradición historiográfica ha datado como hispanomusulmán y que la aplicación del método propuesto en su estudio constructivo ha reconsiderado su datación entre los siglos xiv-Xv. Levantamiento de Ignacio Javier Gil Crespo (2).

Con este método se pretende replantear el conflicto sobre la datación y el estudio constructivo de la arquitectura bajomedieval bajo el punto de vista de la Historia de la Construcción.

Por lo tanto, se definen una serie de herramientas conceptuales fundamentales como la historiografía o la taxonomía - como ciencia de la clasificación- pertenecientes a diversas disciplinas y que confluyen en el estudio histórico, constructivo y arquitectónico de los elementos objeto de análisis en esta investigación. Estas herramientas se complementan con otras que se pueden denominar operativas como el estudio bibliográfico, el análisis estratigráfico de fábricas (7) (8) o las herramientas de laboratorio para la caracterización de materiales. El trabajo arqueológico emplea la arqueometría como herramienta que aglutina los métodos de análisis de materiales y de técnicas constructivas y cuyo objetivo es estudiar las medidas y la naturaleza de los objetos arqueológicos (9).
Análogamente se puede definir la arquimetría o la métrica de la arquitectura como la disciplina que combina el conocimiento analítico de los materiales con sus características físicas, dimensionales e histórico-constructivas con el objeto de su completo conocimiento y caracterización físico-química e histórico-constructiva que permitan situar la fábrica o el edificio que se esté analizando dentro de un período histórico o un grupo constructivo concretos.

Se ha recurrido a la fuente primaria de información, esto es: el edificio. El aprendizaje desde la didáctica de la ruina y su interpretación constructiva conforman el método básico de trabajo. El análisis de los procesos constructivos se lleva a cabo mediante la realización de modelos gráficos como método de interpretación y análisis racional de la arquitectura acompañado de la catalogación geográfica y tipológica, la fotogrametría y la mensiocronología. 


\section{ESTADO DE LA CUESTIÓN}

El análisis constructivo de los restos conservados en las fortificaciones objeto de estudio permite definir una serie de parámetros útiles para la datación de las fábricas. En este sentido, ya hay proyectos de investigación en otras universidades españolas e internacionales que tratan de estudiar métodos de interpretación de las fábricas históricas, además del ya habitual análisis estratigráfico (10).

La arquitectura de tierra, tanto en fábricas históricas como de factura tradicional, ha sido en diversas ocasiones clasificada atendiendo a distintos criterios. Las últimas y más relevantes son las de Maldonado Ramos (11), Maldonado y Vela (12) (13), Tabales (14), Jaquin, Augarde y Gerrard (15), Graciani y Tabales (16) y Mileto, Vegas y García (17).

Las clasificaciones de Maldonado (11) y Maldonado y Vela (12) (13) atienden principalmente a casos de la arquitectura tradicional y entienden las tapias como monolíticas y mixtas, considerando la presencia de otros materiales que refuerzan los cajones de tierra.

Tabales (14) realiza una clasificación básica morfológica y atiende al grado de utilización de la cal, pero no entra a valorar cuestiones métricas. Como él mismo indica, se trata de una clasificación preliminar en doce tipos distintos cuya validez debe ser ratificada con el uso. La clasificación es sistematizada años más tarde realizando una clasificación cronotipológica por Graciani y Tabales (16) y se tiene en cuenta la distinción entre tapias monolíticas y mixtas y realiza una completa clasificación de estas últimas en cuanto a forma, métrica y construcción. Por último señala las épocas históricas de uso preferente de cada fábrica (pre-almohade, almohade, mudéjar, Edad Moderna y Edad Contemporánea). Esta amplia sistematización se refiere al ámbito sevillano.

Jaquin, Augarde y Gerrard (15), mediante el estudio de 60 edificios construidos en tapia de tierra entre 967 y 1837, repartidos por el sureste peninsular y la provincia de Zaragoza, realizan una caracterización histórica de la técnica y una clasificación en diez tipos distintos.

Por último, Mileto, Vegas y García (17) ofrecen un acercamiento general a las tapias de tierra de la arquitectura fortificada española. Este estudio supera la dimensión regional para establecer unos criterios de análisis de mayor envergadura: desde unos invariantes de materiales se establece una clasificación en subcategorías para cada caso que definen los variantes que incluyen los refuerzos de hiladas o esquinas, empleando los términos de los autores. La toma de datos se ha realizado fundamentalmente por el sureste español.

Todas estas clasificaciones buscan ordenar las fábricas de tapia desde distintos tipos construidos. Al contemplar cada tipo la totalidad de la fábrica, el número de excepciones puede ser mayor que el de tipos fundamentales. Por este motivo se propone a continuación una nueva clasificación taxonómica, tipológico-constructiva y alfanumérica que contempla todos los elementos constructivos referentes a la tapia de tierra como técnica constructiva, material, compactación, función estructural, encofrado, acabado, combinaciones con otros materiales y mechinales en cuanto a su situación, profundidad, formación y sección.

\section{ANÁLISIS Y RESULTADOS}

\subsection{Documentación y toma de datos}

Las fábricas de tapia de tierra y mampostería encofrada documentadas se localizan principalmente en el ámbito castellano, aragonés, andaluz y el mediodía portugués (Figura 2). Estas fábricas corresponden a construcciones militares medievales, tanto hispanomusulmanas como de factura cristiana. La toma de datos para el estudio de la construcción encofrada en la fortificación medieval se ha realizado en diversas campañas y se ha documentado un significativo número de castillos, alcazabas, torres o murallas construidos tanto en tapia de tierra como en tapia de cal y canto o mampostería encofrada. La distribución geográfica de cada edificio se ha realizado mediante una aplicación de Google Maps que permite la ubicación de cada elemento de estudio y su caracterización y catalogación a través de una ficha descriptiva y su ordenación en capas que incluyen las diversas técnicas constructivas. A pesar de que el número de casos de estudio es significativo, el análisis se ha completado con datos de otras fábricas pertenecientes a otras regiones (como es el caso de las torres hispanomusulmanas valencianas) tomados de referencias bibliográficas (18) (19) (20) (21).
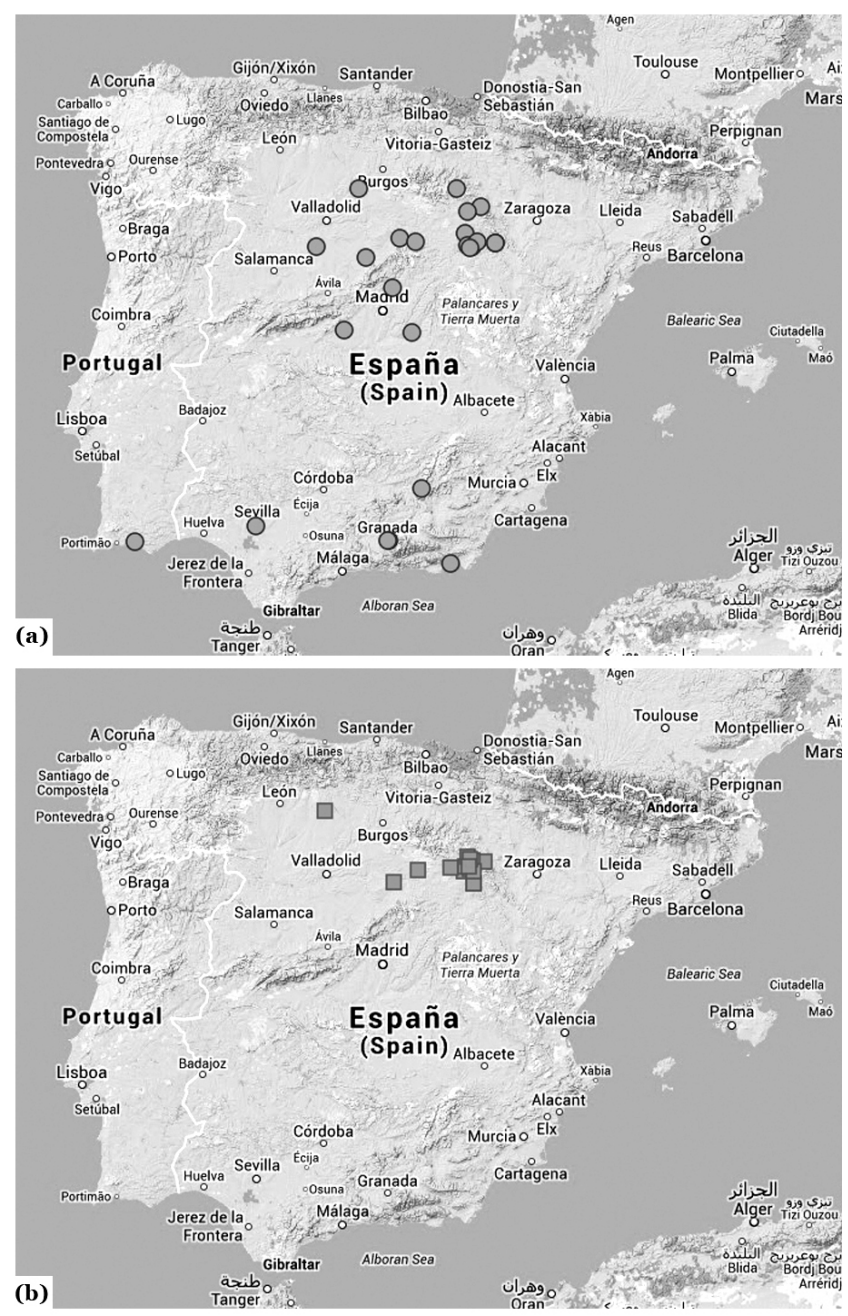

Figura 2. Fábricas de tapia de tierra (a) y fábricas de mampostería encofrada o tapia de cal y canto (b) documentadas en la Península Ibérica (elaboración propia sobre Google Maps). 
En cada una de las fábricas documentadas se han recopilado datos dimensionales y constructivos con el fin de comparar los datos en función de los mismos parámetros de análisis y con relación cronológica. Estos datos sobre las dimensiones de la fábrica de tapia y mechinales registradas durante el proceso de toma de datos son los siguientes: h: altura del nivel constructivo; e: espesor del muro; $l$ : longitud de los cajones; $\alpha$ : talud de las juntas constructivas; $d$ : distancia entre mechinales; $x$ : anchura del mechinal; $y$ : altura del mechinal; $p$ : profundidad del mechinal.

La Tabla 1 recoge los datos tomados en una serie de fábricas de tapia de tierra en algunas construcciones fortificadas representativas de los períodos hispanomusulmán (califal, taifal, almohade y nazarí) y bajomedieval cristiano.

\subsection{Taxonomía constructivo-tipológica}

La clasificación constructivo-tipológica que se propone en esta investigación atiende a las diversas maneras de construcción de las fábricas denominadas genéricamente como encofradas o de tapia. En base a criterios constructivos se ha elaborado una clasificación taxonómica que recoge diversos parámetros constructivos de las fábricas. El primer parámetro, denominado TA, diferencia las tapias según el material que se introduce dentro del encofrado: tierra, tierra con tongadas de cal, tierra hormigonada (tierra con cal y rebles, ripios o cascotes), cal y canto o mampostería. El segundo parámetro de análisis (TB) fija la compactación del material, ya que éste se puede introducir dentro del encofrado y compactarlo con un pisón (TB1) o se puede simplemente verter para su posterior fraguado (TB2).

En tercer lugar, se recoge la función constructiva de la fábrica de tapia. En este caso se diferencia entre la fábrica principal
(TC1), el relleno de paramentos ( $\mathrm{TC}_{2}$ ) o el relleno interior de un muro de piedra o de ladrillo ( $\left.\mathrm{TC}_{3}\right)$.

El cuarto punto de estudio es la forma del encofrado, si es de cajones independientes con tapiales testeros o fronteras (TD1) o si por el contrario se hacen hilos continuos con junta inclinada (TD2). También se diferencian aquellas tapias que se construyen como relleno de los cuarterones entre machones de ladrillo o piedra (TD3)

El quinto aspecto reseñado es el acabado superficial, que contempla los casos de la tierra apisonada sin revestimiento (TE1), una capa calicostrada (TE2), la tierra sin acabado (TE3), una capa externa de sillería (TE4) o de mampostería (TE5).

El sexto nivel de comparación consiste en los materiales complementarios que puede tener una fábrica de tapia de tierra: si sólo es tierra ( $\mathrm{TF} 1)$, si aparecen verdugadas de ladrillo (TF2), brencas de cal ( $\left.\mathrm{TF}_{3}\right)$ y otros ( $\left.\mathrm{TF}_{4}\right)$.

La situación, profundidad, formación y sección de los mechinales configuran el resto de parámetros de análisis de la fábrica de tapia (TGa-TGb-TGc-TGd). La situación de los mechinales puede ser en el propio hilo (TGa1), cajeados en el hilo inferior (TGa2), en el espesor de la verdugada de ladrillo (TGa3) o fuera de la fábrica encofrada o de tapia (TGa4).

Las profundidad de los mechinales de los agujales indica que se emplearon medias agujas (TGb1) o agujas pasantes (TGb2), lo que establece otro nivel de comparación. Por último, es fundamental atender a la formación de los mechinales. El más sencillo es el que deja la aguja embebida en la fábrica (TGc1), con su posterior pudrición y desaparición si bien en muchos casos se ha llegado a conservar

Tabla 1. Estudio dimensional de las fábricas de tapia de tierra en una selección de fortificaciones.

\begin{tabular}{|c|c|c|c|c|c|c|c|c|c|}
\hline \multirow[b]{2}{*}{ Edificio } & \multirow[b]{2}{*}{ Elemento } & \multirow{2}{*}{$\begin{array}{c}\text { Fecha de } \\
\text { construcción }\end{array}$} & \multicolumn{4}{|c|}{ Dimensiones de la tapia } & \multicolumn{3}{|c|}{ Mechinal } \\
\hline & & & $\boldsymbol{h}$ & $\boldsymbol{e}$ & $l$ & $\begin{array}{c}d \\
\text { (mechinales) }\end{array}$ & $x$ & $y$ & $p$ \\
\hline Ayllón (SG) & Castillo & siglos X-XI & $90-100$ & $250-280$ & $200-220$ & $50-70$ & $12-15 ; \emptyset 12$ & $7-10-15$ & 70 \\
\hline Caracena (SO) & Coracha & ¿siglos X-XI? & 80 & $150 ?$ & continuo & 100 & 3 & 7 & no pasantes \\
\hline Paderne, Portugal & Castillo & siglo XII & $85-90$ & $\pm 2 \mathrm{~m}$ & 330 & $80-85$ & 2 & 6 & no pasantes \\
\hline Sevilla (SE) & Murallas & siglos XII-XIII & $80-90$ & & $240-250$ & $82-85$ & $82-85$ & 60 & $5,5 \times 5,5 ; 2 \times 6$ \\
\hline Almería (AL) & Alcazaba & siglo XIII & 90 & & 180 & $85-90$ & 5 & 2 & no pasantes \\
\hline Medina del Campo (VA) & $\begin{array}{l}\text { Castillo de } \\
\text { La Mota }\end{array}$ & siglo XIV & $\begin{array}{c}150+15- \\
20\end{array}$ & ? & $\begin{array}{l}\text { entre machones } \\
\text { de ladrillo }\end{array}$ & variable & & & \\
\hline Palenzuela (P) & Castillo & siglo XV & 110 & 270 & continuo & $85-90$ & varios $7,5-15$ & varios 9-17 & pasantes \\
\hline Serón de Nágima (SO) & Castillo & siglos XIV-XV & 90 & 270 & continuo & 70 & $7-10$ & $14-10$ & pasantes \\
\hline Yanguas (SO) & Castillo & siglos XIV-XV & 125 & $180 ?$ & 300-variable & $70-100-120$ & $6-8$ & $6-8$ & no pasantes \\
\hline Embid de Ariza (Z) & Coracha & siglo XIV & 110 & 90 & $\begin{array}{l}\text { continuo/ } \\
\text { adaptado a la } \\
\text { pendiente }\end{array}$ & $90-100$ & 12,5 & 17 & pasantes \\
\hline Monreal de Ariza (Z) & Castillo & siglos XII-XIV & 110 & $220-240+8$ & continuo & $85-90$ & $8-10$ & $10-17$ & pasantes \\
\hline Turégano (SG) & $\begin{array}{l}\text { Cerca } \\
\text { del castillo }\end{array}$ & siglos XIV-XV & 110 & $180-210$ & $290-315$ & $75-80-90$ & $3,5-5-10-12$ & $8-9-14-15$ & pasantes \\
\hline Fuentidueña de Tajo (M) & Castillo & siglos XIV-XV & $100-105$ & 270 & 270 & $80-84$ & 6 & 11 & pasantes \\
\hline $\begin{array}{l}\text { Castillo de La Raya, } \\
\text { Monteagudo de las } \\
\text { Vicarías (SO) }\end{array}$ & Torreón & siglo XIII-XIV & 120 & ? & ? & $?$ & ? & $?$ & pasantes \\
\hline
\end{tabular}


el elemento de madera. Otros mechinales, en los que se pretende recuperar las agujas para su reutilización, son los que forman un cielo de mampuestos o ladrillos (TGc2), un cajeado de ladrillo con paredes internas formadas también de piezas latericias ( $\mathrm{TGc}_{3}$ ) o un cajeado realizado con la interrupción de la verdugada de ladrillo entre hilo e hilo de tierra ( $\left.\mathrm{TGc}_{4}\right)$.

Por último, se diferencian las escuadrías de las agujas de los tapiales entre aquéllas de sección cuadrangular (TGd1), rectangular (TGd2), plana (TGd3), circular (TGd4), varillas pareadas (TGd5) y de sector de circunferencia (TGd6).
La Tabla 2 recopila todas las fábricas encofradas estudiadas en la investigación y su clasificación según los criterios explicados. Este sistema de clasificación permite codificar y ordenar las distintas fábricas de manera sistemática y completa, pudiéndose extraer tipos constructivos parciales para cada elemento o parámetro de control y generales de la fábrica. Posee la virtud, además, de ser un sistema de categorización abierto: permite la ampliación ante la eventual aparición de una nueva variante o elemento no contemplado como parámetro de control (Figura 3).

Este sistema clasificatorio permite establecer comparaciones entre las distintas fábricas independizando cada uno de los

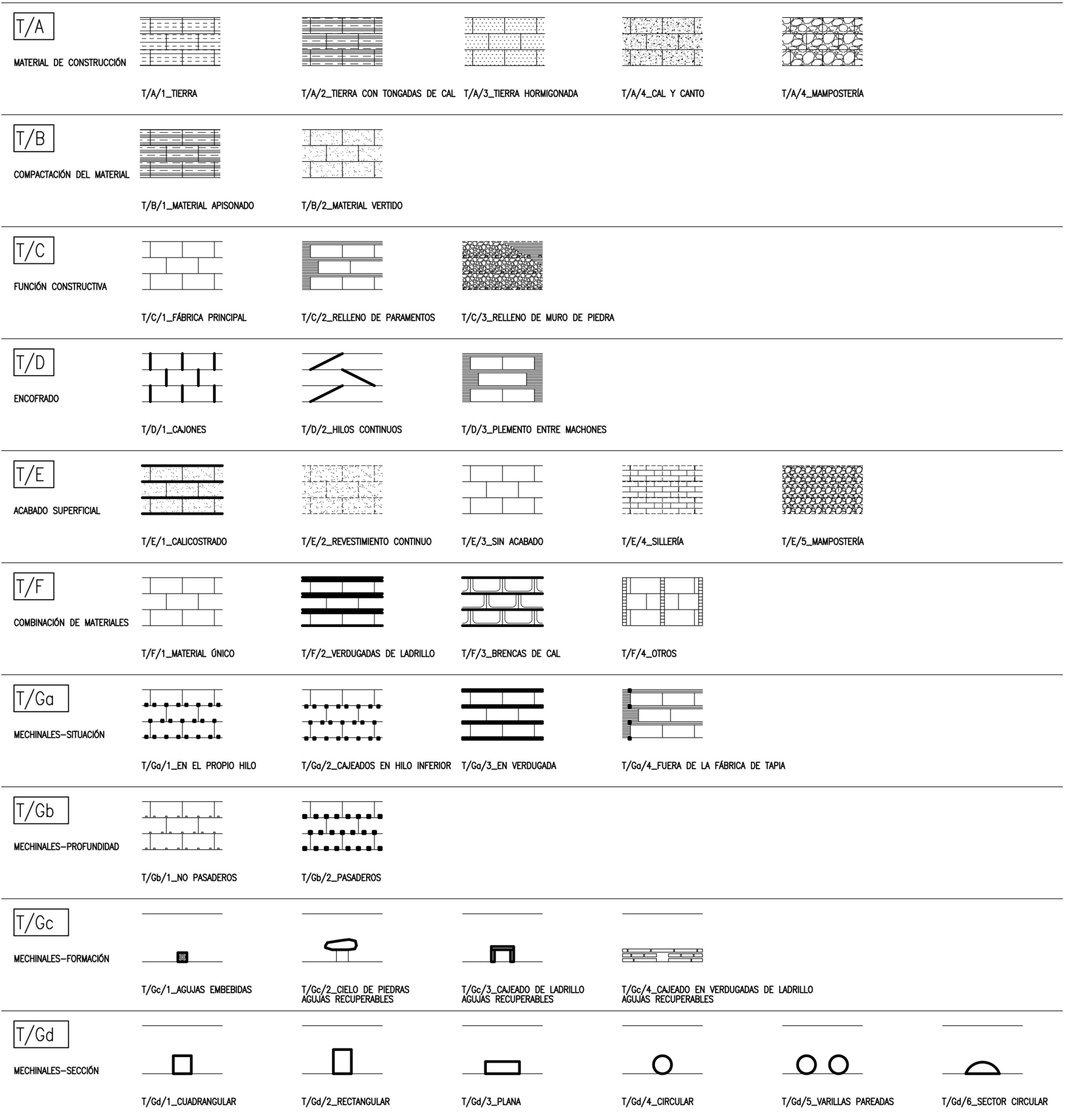

Figura 3. Taxonomía constructiva de las fábricas encofradas. Parámetros con los que se han analizado las fábricas de tapia de tierra o de cal y canto de las fortificaciones estudiadas para la presente investigación. 
Tabla 2. Cronotipología constructiva de las fábricas encofradas estudiadas en la presente investigación.

\begin{tabular}{|c|c|c|c|c|c|c|c|c|c|c|c|c|}
\hline \multirow{3}{*}{ Localidad } & \multirow{3}{*}{ Elemento } & \multirow{3}{*}{ Datación } & \multicolumn{10}{|c|}{ Taxonomía constructiva } \\
\hline & & & \multirow{2}{*}{$\begin{array}{c}\text { A. } \\
\text { Material }\end{array}$} & \multirow{2}{*}{$\begin{array}{c}\text { B. } \\
\text { Compactación }\end{array}$} & \multirow{2}{*}{\begin{tabular}{|c|}
$\mathrm{C}$. \\
Función cons- \\
tructiva
\end{tabular}} & \multirow{2}{*}{$\begin{array}{c}\text { D. } \\
\text { Encofrado }\end{array}$} & \multirow{2}{*}{\begin{tabular}{|c|} 
E. \\
Acabado \\
superficial
\end{tabular}} & \multirow{2}{*}{\begin{tabular}{|c|}
$\mathrm{F}$. \\
Combinación \\
de materiales
\end{tabular}} & \multicolumn{4}{|c|}{ G. Mechinales } \\
\hline & & & & & & & & & \begin{tabular}{c|} 
Ga. \\
Situación
\end{tabular} & \begin{tabular}{c|} 
Gb. \\
Profundidad
\end{tabular} & \begin{tabular}{c|} 
Gb. \\
Formación
\end{tabular} & $\begin{array}{c}\text { Gc. } \\
\text { Sección }\end{array}$ \\
\hline $\operatorname{Ariza}(\mathrm{Z})$ & Castillo & iIX-X? & TA1 & $\mathrm{TB} 1$ & $\mathrm{TC} 1$ & TD1 & TE1 & $\mathrm{TF} 1$ & TGa1 & TGb1 & TGc1 & $\mathrm{TGd}_{3} / 4$ \\
\hline Calatayud (Z) & $\begin{array}{l}\text { Murallas/Castillo } \\
\text { de la Torre Mocha }\end{array}$ & IX-X & TA1 & TB1 & $\mathrm{TC} 1$ & $\mathrm{TD} 2$ & $\mathrm{TE}_{3}$ & $\mathrm{TF}_{3}$ & TGa1 & TGb1 & TGc1 & \\
\hline Calatayud (Z) & Castillo de Ayub & IX-X & $\mathrm{TA}_{3}$ & TB2 & $\mathrm{TC} 1$ & TD1 & $\mathrm{TE}_{3}$ & TF1 & & & & \\
\hline $\begin{array}{l}\text { Fuentidueña } \\
\text { de Tajo (M) }\end{array}$ & Albacar & $x-X I$ & $\mathrm{TA}_{3}$ & TB1 & $\mathrm{TC} 1$ & & TE1 & $\mathrm{TF} 1$ & TGa1 & TGb1 & TGc1 & TGd4 \\
\hline Ayllón (SG) & Castillo & $\mathrm{X}$-XI & TA1 & $\mathrm{TB} 1$ & $\mathrm{TC} 1$ & TD1 & TE1 & $\mathrm{TF} 1$ & TGa2 & TGb1 & TGc2 & $\mathrm{TGd} 2 / 3 / 4$ \\
\hline Caracena (SO) & Coracha & $\mathrm{X}$-XI? & TA1 & TB1 & $\mathrm{TC}_{3}$ & TD1 & $\mathrm{TE}_{5}$ & $\mathrm{TF} 1$ & TGa1 & TGb1 & $\mathrm{TGc1}$ & $\mathrm{TGd} 3$ \\
\hline Castellanos (S0) & Torreón & $\mathrm{X}$-XI? & TA4 & $\mathrm{TB} 2$ & $\mathrm{TC} 1$ & TD2 & $\mathrm{TE}_{3}$ & $\mathrm{TF1}$ & TGa1 & TGb1 & TGc1 & \\
\hline $\begin{array}{l}\text { Hinojosa del } \\
\text { Campo (S0) }\end{array}$ & $\begin{array}{l}\text { Torreón / } \\
\text { Campanario }\end{array}$ & ix-XI? & $\mathrm{TA}_{4}$ & TB2 & $\mathrm{TC} 1$ & TD2 & $\mathrm{TE}_{3}$ & $\mathrm{TF} 1$ & TGa1 & TGb1 & $\mathrm{TGc1}$ & $\mathrm{TGd}_{3} / 5$ \\
\hline La Pica (SO) & Torreón & ¿x-XI? & TA4 & $\mathrm{TB} 2$ & $\mathrm{TC} 1$ & $\mathrm{TD} 2$ & $\mathrm{TE}_{3}$ & $\mathrm{TF1}$ & TGa1 & TGb1 & $\mathrm{TGC1}$ & \\
\hline Masegoso (S0) & Torreón & ¿x-Xx? & $\mathrm{TA}_{4}$ & TB2 & $\mathrm{TC} 1$ & TD2 & $\mathrm{TE}_{3}$ & TF1 & TGa1 & TGb1 & TGc1 & \\
\hline $\begin{array}{l}\text { Montenegro } \\
\text { de Ágreda (S0) }\end{array}$ & Torreón & ¿x-xx? & $\mathrm{TA}_{4}$ & $\mathrm{~TB} 2$ & $\mathrm{TC} 1$ & $\mathrm{TD} 2$ & $\mathrm{TE}_{3}$ & $\mathrm{TF} 1$ & TGa1 & TGb1 & TGc1 & \\
\hline Noviercas (S0) & Torreón & ¿X-XI? & TA5 & TB2 & $\mathrm{TC}_{1}$ & TD2 & $\mathrm{TE}_{3}$ & $\mathrm{TF} 1$ & TGa1 & TGb1 & TGc1 & \\
\hline Trébago (S0) & Torreón & ix-XI? & TA5 & TB2 & $\mathrm{TC} 1$ & TD2 & $\mathrm{TE}_{3}$ & $\mathrm{TF} 1$ & TGa1 & TGb1 & TGc1 & \\
\hline Ágreda (S0) & Torreón de La Muela & ix-XI? & TA5 & TB2 & $\mathrm{TC} 1$ & TD2 & $\mathrm{TE}_{3}$ & TF1 & TGa1 & TGb1 & TGc1 & \\
\hline Granada (GR) & $\begin{array}{l}\text { Alcazaba de la } \\
\text { Alhambra }\end{array}$ & XII & $\mathrm{TA}_{3}$ & $\mathrm{~TB} 1$ & $\mathrm{TC} 1$ & $\mathrm{TD} 1$ & TE1 & $\mathrm{TF} 1$ & TGa1 & TGb1 & TGc1 & $\mathrm{TGd}_{3} / 4$ \\
\hline $\begin{array}{l}\text { Castillejo de } \\
\text { Robledo (S0) }\end{array}$ & Castillo & XII & $\mathrm{TA}_{4}$ & $\mathrm{~TB} 1$ & $\mathrm{TC} 1$ & $\mathrm{TD} 1$ & TE1 & $\mathrm{TF} 1$ & TGa1 & TGb2 & $\mathrm{TGc2}$ & $\mathrm{TGd} 2$ \\
\hline La Iruela (J) & Castillo & XII & $\mathrm{TA}_{3}$ & $\mathrm{~TB} 1$ & $\mathrm{TC} 1$ & $\mathrm{TD} 1$ & TE1 & TF1 & TGa1 & TGb1 & TGc1 & TGd4 \\
\hline $\begin{array}{l}\text { Paderne, } \\
\text { Portugal }\end{array}$ & Castillo & XII & $\mathrm{TA} 3$ & $\mathrm{~TB} 1$ & $\mathrm{TC} 1$ & TD1 & $\mathrm{TE} 1 / 2$ & $\mathrm{TF} 1$ & TGa1 & TGb1 & TGc1 & TGd3 \\
\hline Peñalcázar (SO) & Murallas muro M1 & XII & $\mathrm{TA}_{4}$ & TB2 & $\mathrm{TC}_{3}$ & & $\mathrm{TE}_{5}$ & TF1 & TGa1 & TGb1 & & TGd1 \\
\hline Peñalcázar (SO) & Murallas muro M2 & XII & $\mathrm{TA}_{4}$ & TB2 & $\mathrm{TC} 1$ & TD1 & $\mathrm{TE}_{3}$ & $\mathrm{TF} 1$ & TGa1 & TGb1 & TGc1 & $\mathrm{TGd} 1 / 4$ \\
\hline Peñalcázar (S0) & Murallas muro $\mathrm{M}_{3}$ & XII & $\mathrm{TA} 4 / 5$ & TB2 & $\mathrm{TC}_{1}$ & TD1 & $\mathrm{TE}_{3}$ & TF1 & TGa1 & TGb1/2 & TGc1 & $\mathrm{TGd} 1 / 2$ \\
\hline Peñalcázar (S0) & Murallas torre $\mathrm{T} 1$ & XII & $\mathrm{TA}_{4}$ & TB2 & $\mathrm{TC}_{1}$ & TD1 & $\mathrm{TE}_{3}$ & TF1 & TGa1 & TGb1 & TGc1 & $\mathrm{TGd} 4 / 5$ \\
\hline $\begin{array}{l}\text { Peroniel del } \\
\text { Campo (SO) }\end{array}$ & Castillo & XII & $\mathrm{TA} 4 / 4$ & TB2 & $\mathrm{TC} 1$ & $\mathrm{TD} 1$ & $\mathrm{TE}_{3}$ & $\mathrm{TF} 1$ & TGa1 & $\mathrm{TGb1} / 2$ & $\mathrm{TGc1}$ & $\mathrm{TGd} 4 / 5$ \\
\hline Saldaña (P) & Castillo & XII & $\mathrm{TA}_{4}$ & TB2 & $\mathrm{TC}_{3}$ & & $\mathrm{TE}_{5}$ & $\mathrm{TF} 1$ & & & & \\
\hline Soria (S0) & Castillo & XII & $\mathrm{TA}_{4}$ & TB2 & $\mathrm{TC} 1$ & TD1 & $\mathrm{TE}_{3}$ & $\mathrm{TF} 1$ & TGa1 & TGb1 & TGc1 & $\mathrm{TGd} 3$ \\
\hline $\begin{array}{l}\text { Vozmediano } \\
\text { (S0) }\end{array}$ & Castillo & XII & $\mathrm{TA} 4 / 5$ & TB2 & $\mathrm{TC} 1$ & TD1 & $\mathrm{TE}_{3}$ & $\mathrm{TF} 1$ & TGa1 & TGb1 & $\mathrm{TGc1}$ & TGd2 \\
\hline Sevilla (SE) & Murallas & XII-XIII & $\mathrm{TA} 1$ & $\mathrm{~TB} 1$ & $\mathrm{TC} 1$ & TD1 & $\mathrm{TE}_{3}$ & $\mathrm{TF} 1$ & TGa1 & TGb1 & TGc1 & $\mathrm{TGd} 3$ \\
\hline Almería (AL) & Alcazaba & XIII & $\mathrm{TA}_{3}$ & TB1 & $\mathrm{TC} 1$ & TD1 & TE1 & TF2 & $\mathrm{TGa3}$ & TGb1 & $\mathrm{TGC4}$ & TGd1 \\
\hline $\begin{array}{l}\text { Castillo de } \\
\text { La Raya (SO) }\end{array}$ & Torreón & XIII-XIV & TA1 & $\mathrm{TB} 1$ & $\mathrm{TC}_{3}$ & & $\mathrm{TE}_{5}$ & $\mathrm{TF} 1$ & & & $\mathrm{TG} 22$ & TGd2 \\
\hline $\begin{array}{l}\text { Embid } \\
\text { de Ariza (Z) }\end{array}$ & Coracha & $\mathrm{XIV}$ & $\mathrm{TA}_{3}$ & TB2 & $\mathrm{TC} 1$ & $\mathrm{TD} 1$ & TE1 & TF1 & TGa2 & TGb2 & $\mathrm{TGc2}$ & TGd2 \\
\hline Escalona (T0) & Castillo & $\mathrm{XIV}$ & $\mathrm{TA}_{3}$ & TB2 & $\mathrm{TC}_{2}$ & $\mathrm{TD}_{3}$ & $\mathrm{TE}_{3}$ & TF2 & $\mathrm{TGa} 3$ & & $\mathrm{TGC4}$ & TGd1 \\
\hline Granada (GR) & Muralla nazarí & $\mathrm{XIV}$ & $\mathrm{TA} 3$ & $\mathrm{~TB} 1$ & $\mathrm{TC} 1$ & TD1 & TE1 & $\mathrm{TF} 1$ & TGa1 & TGb1 & TGc1 & TGd3 \\
\hline $\begin{array}{l}\text { Medina del } \\
\text { Campo (VA) }\end{array}$ & Castillo de La Mota & $\mathrm{XIV}$ & TA1 & TB2 & $\mathrm{TC} 2$ & $\mathrm{TD}_{3}$ & $\mathrm{TE}_{3}$ & TF2 & $\mathrm{TGa} 3 / 4$ & TGb1 & $\mathrm{TGC4}$ & TGd1 \\
\hline $\begin{array}{l}\text { Monreal } \\
\text { de Ariza (Z) }\end{array}$ & Castillo & XII-XIV & TA1 & $\mathrm{TB} 1$ & $\mathrm{TC} 1$ & TD2 & TE1 & $\mathrm{TF} 1$ & TGa2 & TGb2 & $\mathrm{TGC2}$ & TGd2 \\
\hline $\begin{array}{l}\text { Talamanca } \\
\text { de Jarama (M) }\end{array}$ & Murallas & XII-XIV & $\mathrm{TA} 1$ & $\mathrm{~TB} 1$ & $\mathrm{TC} 1 / 2$ & $\mathrm{TD} 2 / 3$ & $\mathrm{TE}_{3}$ & $\mathrm{TF} 1$ & TGa1 & & $\mathrm{TGc1}$ & $\mathrm{TGd} 1 / 4 / 6$ \\
\hline Turégano (SG) & Cerca del castillo & $\mathrm{XIV}-\mathrm{XV}$ & TA1 & $\mathrm{TB} 1$ & $\mathrm{TC} 1$ & $\mathrm{TD} 1$ & TE1 & $\mathrm{TF} 1$ & TGa2 & TGb2 & $\mathrm{TGC2}$ & $\mathrm{TGd} 2 / 3 / 6$ \\
\hline \begin{tabular}{l|} 
Villafranca \\
del Castillo (M)
\end{tabular} & Castillo de Aulencia & $\mathrm{XIV}-\mathrm{XV}$ & $\mathrm{TA}_{3}$ & $\mathrm{~TB} 2$ & $\mathrm{TC}_{2}$ & $\mathrm{TD} 1 / 3$ & $\mathrm{TE}_{3}$ & $\mathrm{TF} 2$ & TGa3 & TGb1 & $\mathrm{TGC4}$ & TGd1 \\
\hline $\begin{array}{l}\text { Fuentidueña } \\
\text { de Tajo (M) }\end{array}$ & Castillo & $\mathrm{XIV}-\mathrm{XV}$ & $\mathrm{TA}_{3}$ & $\mathrm{~TB} 1$ & $\mathrm{TC} 1$ & $\mathrm{TD} 1$ & TE1 & $\mathrm{TF} 1$ & TGa2 & TGb2 & $\mathrm{TGc2}$ & $\mathrm{TGd} 2$ \\
\hline $\begin{array}{l}\text { Serón de } \\
\text { Nágima (SO) }\end{array}$ & $\begin{array}{l}\text { Arranque } \\
\text { de los muros }\end{array}$ & $\mathrm{XIV}-\mathrm{XV}$ & $\mathrm{TA} 1 / 3$ & $\mathrm{~TB} 1$ & $\mathrm{TC} 1$ & $\mathrm{TD} 2$ & TE1 & $\mathrm{TF} 1$ & $\mathrm{TGa2}$ & TGb2 & $\mathrm{TGc2}$ & $\mathrm{TGd} 1 / 2$ \\
\hline $\begin{array}{l}\text { Serón de } \\
\text { Nágima (SO) }\end{array}$ & $\begin{array}{l}\text { Parte superior } \\
\text { de los muros }\end{array}$ & $\mathrm{XIV}-\mathrm{XV}$ & TA1 & TB1 & $\mathrm{TC}_{1}$ & TD2 & $\mathrm{TE}_{4}$ & $\mathrm{TF} 1$ & TGa2 & TGb2 & $\mathrm{TGC2}$ & $\mathrm{TGd} 1 / 2$ \\
\hline Yanguas (S0) & Castillo & $\mathrm{XIV}-\mathrm{XV}$ & $\mathrm{TA}_{3}$ & TB2 & $\mathrm{TC} 1$ & TD1 & $\mathrm{TE}_{3}$ & $\mathrm{TF} 1$ & TGa1 & TGb1 & TGc1 & TGd1 \\
\hline Palenzuela (P) & Castillo & $\mathrm{xv}$ & $\mathrm{TA} 1 / 3$ & TB1 & $\mathrm{TC} 1$ & TD2 & TE1 & $\mathrm{TF} 1$ & TGa2 & TGb2 & TGc2 & $\mathrm{TGd} 1 / 2$ \\
\hline Magaña (SO) & Castillo & $\mathrm{xv}$ & $\mathrm{TA} 5$ & TB2 & $\mathrm{TC}_{1}$ & TD2 & $\mathrm{TE} 2 / 3$ & $\mathrm{TF} 1$ & TGa1 & TGb1/2 & $\mathrm{TGcl} / 2$ & TGd1 \\
\hline Ucero (S0) & Castillo & $\mathrm{XV}$ & $\mathrm{TA} 5$ & TB2 & $\mathrm{TC} 1$ & TD1 & $\mathrm{TE}_{3}$ & TF1 & TGa1 & TGb1 & TGc1 & $\mathrm{TGd} 1 / 4$ \\
\hline
\end{tabular}



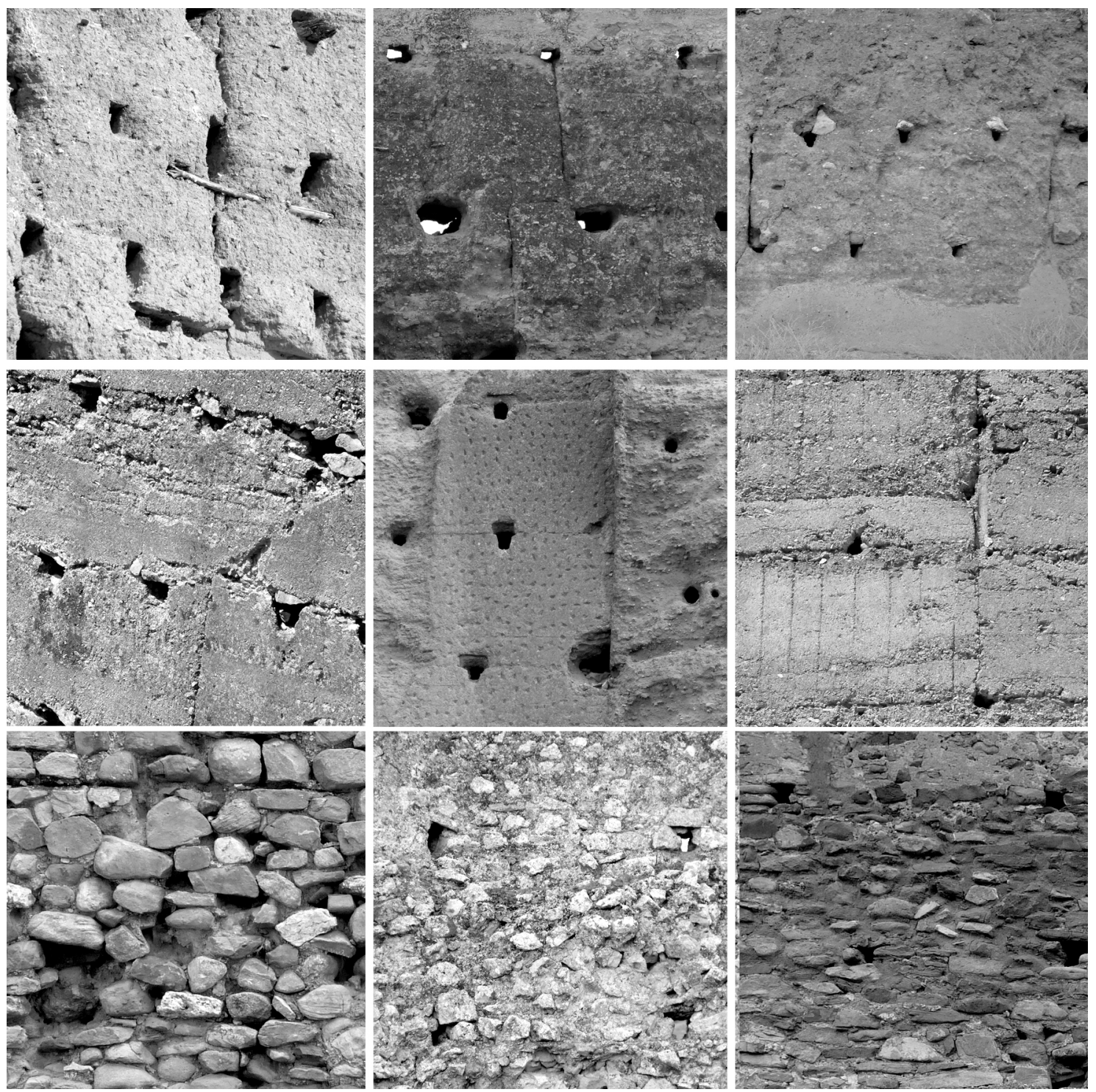

Figura 4. Detalles de las fábricas de tapia de tierra y de de tapia de cal y canto o mampostería encofrada de los castillos y murallas de, Palenzuela (a), Turégano (b), Fuentidueña de Tajo (c), Embid de Ariza (d), Serón de Nágima (e), Yanguas (f), Peroniel del Campo (g), Peñalcázar (h) y Magaña (i).

(Fotografías de Ignacio Javier Gil Crespo).

parámetros de análisis con el fin de establecer grupos constructivos, tipológicos y cronológicos. En base a estos criterios, se pueden clasificar las distintas tapias de tierra estudiadas en la presente investigación.

Una de las ventajas de este sistema de clasificación tipológica de los aspectos constructivos de las fábricas encofradas es que es un sistema abierto, esto es: en caso de aparecer una variante constructiva no recogida entre las subcategorías siempre se podrá añadir los que sean necesarios para que cada fábrica quede completamente caracterizada (Figura 4).

\section{3•3. Arquimetría y grupos cronotipológicos}

A la luz de los datos extraídos del trabajo de campo donde se han documentado un número significativo de fábricas de tapia de tierra y de los datos aportados por los autores consultados (16) (18) (19) (20) (21), se desprende que las tapias hispanomusulmanas tienen unas dimensiones aproximadamente constantes y tienden a estar moduladas según los codos ma’mūní o raššāšś ${ }^{2}$. Las tapias construidas en los estados medievales cristianos españoles tienden a tener unas dimensiones mayores, al menos en altura. La dimensión de $110 \mathrm{~cm}$ de altura de los tapiales se repite sensiblemente para las fortificaciones cristianas, aunque varía entre 95 y $150 \mathrm{~cm}$. En otras construcciones medievales de tapia de tierra fuera de España también se advierte esta constante dimensional, como el caso de las tapias medievales del barrio Saint-

\footnotetext{
${ }^{2}$ Los codos empleados en la arquitectura hispanomusulmana son, principalmente el codo raššăši y el codo mámūní (22). El codo raššăši de 32 dedos, que es la base de la milla y la legua, mide o,55727 m y fue empleado para definir la caña o qas.aba de 6 codos de 32 dedos, conocido más tarde como el Estadal del Consejo Real de Castilla de 12 pies castellanos $-27,86 \mathrm{~cm}-$ y equivalente a $3,3436 \mathrm{~m}$. Hernández (23) da la dimensión de $58,76 \mathrm{~cm}$ y $58,93 \mathrm{~cm}$ para el codo raššāšś. El codo raššāší fue empleado durante el emirato y el califato. Por el contrario, el codo ma'mūní fue la unidad de medida habitual en la construcción almohade y nazarí y se corresponde con o,4714 m para Hernández y o,47 m para Vallbé, mientras que el codo geométrico son $0,4179525 \mathrm{~m}$.
} 
Mathieu de Perpignan, donde las fábricas de las casa de los siglos XIII y XIV tienen unas alturas comprendidas entre los 100 y los $140 \mathrm{~cm}(24)$.

El codo raššāší es mayor que el ma'mūní. La modulación en múltiplos de $55 \mathrm{~cm}$ es propia de la primera unidad de medida, la cual se empleaba durante el emirato y el califato. Con las invasiones almorávide y almohade de los siglos XI y XII se toma como unidad de medida el codo ma'mūní, por lo que la construcción se modula en múltiplos de 45-47 cm.

En la construcción con tapia de tierra castellana, la altura de los tapiales tiende a ser mayor. Desde 90-95 $\mathrm{cm}$ hasta, lo más común, 110-120 cm y otras dimensiones mayores. La altura de niveles de 110 (100-120) $\mathrm{cm}$ es habitual y se repite en otras obras de mampostería, como las murallas de Soria o la parte de mampostería de las de Buitrago. Sin embargo, no se trata de una regla o costumbre general, ya que las divergencias son significativas. Con los datos recogidos se abre el debate acerca del empleo de una modulación en la arquitectura bajomedieval con tapia de tierra (25). La explicación a esta diversidad de módulos o dimensiones quizá se encuentre en la tradición constructiva de los operarios contratados. Sólo un análisis documental -en caso de que se conserven contratos o documentos similares- podrá verificar esta hipótesis (26).

La vara castellana tiene una dimensión de o,8369 m, y el estadal del Consejo Real de Castilla - correspondiente a la caña hispanoárabe de seis codos raššâšíes- equivale a 3,344 m aunque varía según la zona (22). La altura de los tapiales de $110 \mathrm{~cm}$ viene a corresponder con un tercio de estadal o, lo que es lo mismo, dos codos o cuatro pies castellanos de $27,86 \mathrm{~cm}$. En cualquier caso, el análisis de las dimensiones no arroja unos resultados concluyentes, ya que el estado de conservación de las fábricas no permite una toma de datos exacta ni completa.

La separación entre los ejes de los mechinales tampoco arroja resultados concluyentes, pues así como se observa una constancia en la dimensión de este aspecto constructivo en las tapias hispanomusulmanas -las agujas se separan entre 82$85 \mathrm{~cm}-$, en las tapias de construcción cristiana esta medida se reduce a veces a $70 \mathrm{~cm}$ y otras alcanza los $90 \mathrm{~cm}$, por lo que se desprende que no había una intención dimensional en la obra. Incluso dentro de la misma fábrica hay divergencias dimensionales significativas. No obstante, hay casos, como los castillos señoriales de Serón de Nágima (2) o Palenzuela, en los que se aprecia una significativa regularidad en la separación entre los agujales. Esta constancia parece corresponderse con la garantía de una rápida construcción.

Otra de las características de la construcción castellana es que, por lo general, emplea hilos continuos de tapia y no cajones como la hispanomusulmana, además de que los agujales son pasantes, por lo que simplifica el sistema de atado y arriostramiento a base de medias agujas, cuñas, cruces de san Andrés y lías que requieren un trabajo más especializado, además de la fabricación de estos elementos y su abandono en el interior de la fábrica (10) (27) (28). La ventaja de las agujas pasantes estriba en que, además de simplificar su manufactura - tan sólo son vigas escuadradas-, pueden servir de andamiaje volado y ser retiradas y reutilizadas según el avance de la obra.
En lo que respecta a la construcción con tapia de cal y canto, su momento de mayor desarrollo se corresponde con la etapa central de la Edad Media - siglos XII y XIII-, aunque cae en desuso hacia el XIV y xv en beneficio de la construcción de tapia de tierra. El castillo de Peroniel del Campo y las murallas de Peñalcázar, ambas fábricas sitas en la provincia de Soria, tienen similitudes constructivas con los castillos fronterizos entre León y Castilla cuando ambos reinos estuvieron temporalmente separados y enfrentados (1157-1230). En estos últimos se empleó sistemáticamente la fábrica de cal y canto con, en muchos casos, mechinales dobles correspondientes a unas agujas de varillas pareadas (29) (30). Este hecho puede servir de base para una provisional datación de manera que cobra fuerza la hipótesis de que las fortificaciones de la raya oriental de Castilla levantadas con la misma técnica sean coetáneas.

El análisis mensiológico necesita fijar cronológicamente algunos elementos para que por comparación cronotipológica se puedan agrupar los distintos tipos constructivos. La arqueología y las fuentes tienen datadas muchas de las fortificaciones hispanomusulmanas, las cuales revelan un uso de dimensiones y modulaciones constantes basadas en el codo como unidad de medida. El problema surge ante aquellos restos de dudosa que también tienen cierta uniformidad dimensional pero distinta de la anterior y que por el mero hecho de ser una construcción en tierra se ha datado erróneamente como una fortificación musulmana cuando todo apunta a que la construcción cristiana empleaba otros módulos que se han mostrado sensiblemente constantes.

Para la realización de este cometido - la determinación de la datación de ciertas fábricas- se han recogido muestras de agujas contenidas en las fábricas para su posterior datación por radiocarbono o dendrocronología que ponga un límite inferior a la construcción de cada edificio.

Los grupos cronotipológicos propuestos para los tres principales momentos de construcción encofrada en la fortificación medieval ibérica son la fortificación hispanomusulmana, la fortificación castellana de los siglos XII-XIII en tapia de cal y canto y la fortificación cristiana bajomedieval en tapia de tierra. La clasificación cronotipológica propuesta para el primero, que se puede afinar para cada uno de los subgrupos -construcción califal, taifal, almohade y nazarí- es: A1/3, B1, C1/3, D1, E1, F1, Ga1, Gb1, Gc1, Gd3/6. Las características constructivo-morfológicas de la fortificación castrense plenomedieval castellana quedan designadas bajo el código A4/5, B2, C1, D1, E3, F1, Ga1, Gb1/2, Gc1, Gd1/4/5. Por último, la codificación de la fortificación bajomedieval cristiana es, en líneas generales, A1/3, B1/2, C1/2, D2, E1, F1/2, Ga2/3, Gb2, $\mathrm{Bc2} / 4$, Gd2.

Este sistema taxonómico permite atender tanto a grandes grupos constructivos históricos, como los tres antes mencionados, como a ámbitos de estudio más restringidos en los que haya un número significativo de fábricas encofradas. De igual manera, esta clasificación alfanumérica se puede aplicar al estudio de la construcción tradicional con tierra (31).

\section{CONCLUSIONES}

El método empleado en el análisis de las distintas fábricas permite comparar el conjunto de la fábrica y sus elementos de forma independiente en base a los diversos parámetros de 
análisis constructivo y morfológico definidos. Este sistema de análisis cronotipológico, a diferencia de los tradicionalmente empleados como la lectura estratigráfica, es un sistema abierto, de manera que se pueden incluir nuevas categorías que atiendan a toda fábrica para su completa caracterización. Todas las posibles variaciones tipológicas y constructivas están contempladas en el sistema alfanumérico de caracterización constructiva.

El análisis de los fundamentos constructivos del sistema fortificado de frontera ha permitido establecer grupos cronotipológicos que presentan características comunes. No obstante, mediante herramientas propias de la Historia de la Construcción -acompañadas de estudios territoriales, documentales y poliorcéticos, y previas al análisis de caracterización y datación por medios físico-químicos que urge realizar-se pueden establecer grupos constructivos e hitos cronotipológicos en los que estudiar la evolución de cada técnica poniendo en paralelo los valores arquimétricos y los sistemas y medios auxiliares con el fin de ubicar cronotipológicamente cada fábrica y, por ende, cada castillo y fortificación estudiada.

Del análisis de los resultados de esta taxonometría y del análisis dimensional se concluye que se pueden establecer unos grupos cronotipológicos característicos a pesar de que las divergencias dimensionales pueden llegar a ser significativas. Las fábricas de tapia de tierra hispanomusulmanas se modulan sensiblemente en codos y las diferencias dimensionales se deben al tipo de codo empleado en cada época. La presumida modulación de la tapia de tierra de la construcción bajomedieval cristiana parece derivarse de los operarios que la construyen y nace de la adopción de la tradición modular hispanomusulmana que llega a definir el estadal del Consejo
Real de Castilla. En cualquier caso, no se ha llegado a unas conclusiones fuertes derivadas del análisis de las dimensiones debido a la dificultad de tomar datos con exactitud por el mal estado de conservación de las fábricas.

Así como el análisis métrico de la construcción no es capaz de arrojar resultados concluyentes por las divergencias detectadas, el análisis constructivo sí colabora en una embrionaria definición de los grupos constructivos. Las fábricas hispanomusulmanas tienden a construirse las más de las veces con cajones independientes dentro de los cuales se apisona la tierra o tierra mezclada con cal y reble - la tapia militar- y emplea medias agujas que necesitan de un sistema de atado y arriostramiento mediante cuñas, cruces de san Andrés y lías que quedan embebidas dentro de la fábrica. La construcción cristiana, por el contrario, acostumbra a levantar tapias de hilos continuos - si bien hay casos de empleo de cajonessiempre con agujas pasantes, de escuadría generalmente rectangular y de mayor sección, que facilitan y simplifican el trabajo especializado de carpintería auxiliar a la vez que conforman las almojayas reutilizables del andamiaje.

No obstante, estos resultados deben ser considerados como provisionales debido a que el número de casos estudiados es limitado y, aunque con una dispersión geográfica significativa, está circunscrito principalmente al ámbito castellano y de la provincia de Soria. En cualquiera de los grupos propuestos hay excepciones a estas características morfo-constructivas que parecen perfilarse como generales. Con la continuación del análisis incluyendo un mayor número de fábricas históricas pertenecientes a un más amplio ámbito territorial se podrán establecer con mayor precisión diversos grupos cronotipológicos que atiendan a los invariantes morfo-constructivos adecuados a los territorios históricos.

\section{REFERENCIAS}

(1) Gil-Crespo, I. J. (2012). Rammed earth walls in Serón de Nágima castle (Soria, Spain): constructive lecture. En Mileto, C., Vegas, F., Cristini, V. (Eds.), Rammed Earth Conservation (pp. 107-112). London: Taylor \& Francis Group.

(2) Gil-Crespo, I. J. (2013). Fundamentos constructivos de la fortificaciones fronterizas entre las coronas de Castilla y Aragón de los siglos XII al XV en la actual provincia de Soria (Tesis doctoral). Madrid: Universidad Politécnica de Madrid.

(3) Maldonado-Ramos, L., Vela-Cossío, F. (2011). El patrimonio arquitectónico construido con tierra. Las aportaciones historiográficas y el reconocimiento de sus valores en el contexto de la arquitectura popular española. Informes de la Construcción, 63(523): 71-80, doi: http://dx.doi.org/10.3989/ic.10.062.

(4) Schnell-Quiertant, P. (2008-2009). El inventario de arquitectura militar fortificada realizado por la A.E.A.C. Castillos de España, (152-153-154): 1-10.

(5) Cobos-Guerra, F., Retuerce-Velasco, M. (2011). Metodología, valoración y criterios de intervención en la arquitectura fortificada de Castilla y León. Catálogo de las provincias de León, Salamanca, Valladolid y Zamora. Valladolid: Junta de Castilla y León.

(6) Tosco, C. (2003). Il Castello, la casa, la chiesa. Architettura e società nel medioevo. Turín: Einaudi.

(7) Caballero-Zoreda, L., Latorre Gonzalez-Moro, P. (1995). La importancia del análisis estratigráfico de las construcciones históricas en el debate sobre la restauración monumental. Informes de la Construcción, 46(435): 5-18, doi: http:// dx.doi.org/ 10.3989/ic.1995.v46.i435.1093.

(8) Caballero-Zoreda, L. (1995). Método para el análisis estratigráfico de construcciones históricas o «lectura de paramentos». Informes de la Construcción, 46(435): 37-46, doi: http://dx.doi.org/10.3989/ic.1995.v46.i435.1096.

(9) Quirós-Castillo, J. A. (2002). Arqueología de la arquitectura en España. Arqueología de la Arquitectura (1): 27-38.

(10) Graciani-García, A. (2009). Improntas y oquedades en fábricas históricas de tapial. Indicios constructivos. En HuertaFernández, S., Marín, R., Soler, R., Zaragozá, A. (Eds.) Actas del Sexto Congreso Nacional de Historia de la Construcción (Vol. 1, pp. 683-692). Madrid: Instituto Juan de Herrera.

(11) Maldonado-Ramos, L. (1999). Arquitectura construida con tierra en la Comunidad de Madrid. Madrid: Fundación Diego de Sagredo.

(12) Maldonado-Ramos, L., Vela-Cossío, F. (1999). Técnicas y sistemas tradicionales. Curso de Construcción con tierra (I). Madrid: Instituto Juan de Herrera.

(13) Maldonado-Ramos, L., Vela Cossío, F. (1999). Técnicas y sistemas tradicionales. Curso de Construcción con tierra (II). Madrid: Instituto Juan de Herrera. 
(14) Tabales-Rodríguez, M.Á. (2001). Aportaciones de la arqueología medieval al conocimiento de las técnicas constructivas. En A. Graciani-García (Ed.), La técnica de la arquitectura medieval (pp. 35-74). Sevilla: Universidad de Sevilla

(15) Jaquin, P., Augarde, C., Gerrard, C. (2007, 22-24 de agosto). Historic rammed earth structures in Spain, construction techniques and a preliminary classification. En International Symposium on Earthen Structures. Bangalore, India.

(16) Graciani-García, A., Tabales-Rodríguez, M. Á. (2008). El tapial en el área sevillana. Avance cronotipológico estructural. Arqueología de la Arquitectura (5): 135-158.

(17) Mileto, C., Vegas López-Manzanares, F., García-Soriano, L. (2013). La técnica constructiva de la tapia en la arquitectura militar y defensiva en España. Variantes e invariantes. En Huerta-Fernández, S., López-Ulloa, F. S. (Eds.) Actas del Octavo Congreso Nacional de Historia de la Construcción (vol. 2, pp. 681-687). Madrid: Instituto Juan de Herrera.

(18) Rodríguez-Navarro, P. (2008). La torre árabe observatorio en tierras valencianas. Tipología arquitectónica (Tesis doctoral). Valencia: Universidad Politécnica de Valencia.

(19) Mileto, C., Vegas, F., López, J. M. (2011). Criterios y técnicas de intervención en tapia. La restauración de la torre Bofilla de Bétera (Valencia). Informes de la Construcción, 63(523): 81-96, doi: http://dx.doi.org/10.3989/ic.10.014.

(20) López-Osorio, J.M. (2012). The Nasrid ramparts of the Albaicín (Granada, Spain): an analysis of materials and building techniques. En Mileto, C., Vegas, F., Cristini, V. (Eds.) Rammed Earth Conservation (pp. 27-32). London: Taylor \& Francis Group.

(21) Vela-Cossío, F. (2006). Arquitectura y construcción con tierra en fortificaciones musulmanas de la Meseta Norte de España. En Terra: Forma de Construir (pp.138-145). Lisboa: Argumentum.

(22) Vallbé-Bermejo, J. (1976). Notas de metrología hispano-árabe. El codo en la España musulmana. Al-Andalus, Revista de Estudios Árabes de Madrid y Granada, 51(2): 339-354.

(23) Hernández-Giménez, F. (1961). El codo en la historiografía árabe de la mezquita mayor de Córdoba. Madrid: Imprenta y editorial Maestre.

(24) Chazelles, C.-A. d., Guyonnet, F. (2007). La construction en pisé du Languedoc-Roussillon et de la Provence, du MoyenÂge à l'époque moderne (xiI ${ }^{\mathrm{e}}-\mathrm{XIX}^{\mathrm{e}} \mathrm{s}$.). En Guillaud, H., Chazelles, C.-A. d., Klein, A. (Eds.) Les constructions en terre massive: pisé et bauge. Deuxièmes échanges transdisciplinaires sus les constructions en terre crue (pp. 109-139). Villefontaine, Isère: Éditions del'espérou.

(25) Graciani-García, A. (2013). Consideraciones iniciales y reflexiones sobre la tapia como unidad de medida para una interpretación constructiva del término. En Huerta-Fernández, S., López-Ulloa, F. S. (Eds.) Actas del Octavo Congreso Nacional de Historia de la Construcción (Vol. 1, pp. 439-446). Madrid: Instituto Juan de Herrera.

(26) Cómez-Ramos, R. (2006). Los constructores de la España medieval. Sevilla: Universidad de Sevilla. ( $3^{\mathrm{a}}$ ed.: 2009).

(27) Martín-García, M. (2005). La construcción del tapial en época nazarí: el caso de la muralla exterior del Albaicín de Granada. En Huerta-Fernández, S. (Ed.) Actas del Cuarto Congreso Nacional de Historia de la Construcción, Cádiz, 27-29 enero 2005 (vol. 2, pp. 741-748). Madrid: Instituto Juan de Herrera.

(28) Martín-García, M. (2009, 25-27 de marzo). La construcción del tapial calicastrado en época nazarí. En $V$ Convención Técnica de la Arquitectura Técnica (CONTART'o9). Albacete.

(29) Cobos-Guerra, F., Castro-Fernández, J. J. d., Canal Arribas, R. (2012). Castros y recintos de la frontera de León en los siglos XII y XIII. Fortificaciones de tapial de cal y canto o mampostería encofrada. Valladolid: Junta de Castilla y León.

(30) Mañanes-Pérez, T., Valbuena, F. (1977). Torres y fortalezas medievales al sur del Duero en la provincia de Valladolid. Boletín del Seminario de Estudios de Arte y Arqueología (43): 111-126.

(31) Maldonado-Ramos, L., Castilla-Pascual, F. J., Vela-Cossío, F. (1997). La técnica del tapial en la Comunidad autónoma de Madrid. Aplicación de nuevos materiales para la consolidación de muros de tapia. Informes de la Construcción, 49(452): 27-37, doi: http://dx.doi.org/10.3989/ic.1997.v49.i452.925. 